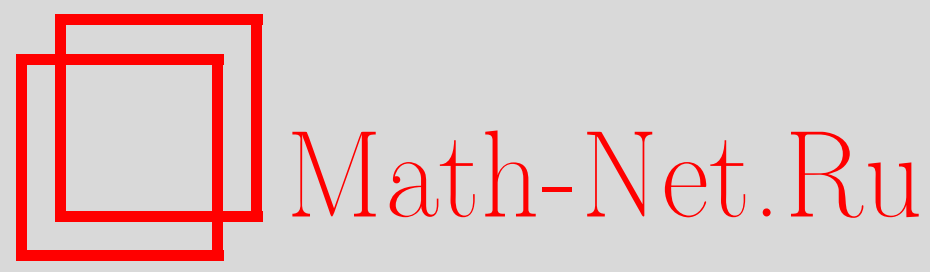

М. Ш. Шабозов, О. Ш. Шабозов, Поперечники некоторых классов аналитических функций в пространстве Харди $H_{2}$, Матем. заметки, 2000, том 68, выпуск 5, 796-800

DOI: https://doi.org/10.4213/mzm1002

Использование Общероссийского математического портала Math-Net.Ru подразумевает, что вы прочитали и согласны с пользовательским соглашением http://www.mathnet.ru/rus/agreement

Параметры загрузки:

IP: 3.85 .183 .62

26 апреля 2023 г., 11:59:39

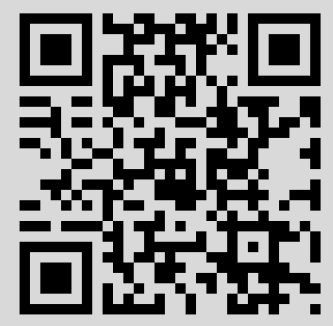




\section{ПОПЕРЕЧНИКИ НЕКОТОРЫХ КЛАССОВ АНАЛИТИЧЕСКИХ ФУНКЦИЙ В ПРОСТРАНСТВЕ ХАРДИ $H_{2}$}

\section{М.Ш. Шабозов, О.Ш. Шабозов}

В последнее время интенсивно изучаются вопросы, связанные с точным вычислением колмогоровских поперечников классов аналитических в единичном круге функций (см. [1]-[3] и библиографические коментарии к ним). С. Б. Вакарчуком [4] в пространстве Харди $H_{2}$ вычислены колмогоровские поперечники некоторых классов аналитических функций, структурные свойства которых характеризуются скоростью убьвания к нулю модуля непрерьвности граничных значений производных функций. Аналогичные результаты для других классов функций получены в [5]. Результаты [4], [5] являются продолжением исследований работ [1]-[3].

Цель настоящей статьи - обобщить результаты [1]-[5] для классов функций, задаваемых модулями непрерывности высших порядков, и найти точные значения различных поперечников навведеных ниже классах функций.

Напомним определение поперечников центрально симмметричного множества $\mathfrak{M}$ в линейном нормированном пространстве $X$ :

$$
\begin{aligned}
& d_{n}(\mathfrak{M}, X)=\inf \left\{\sup \left\{\inf \left\{\|f-\varphi\|_{X}: \varphi \in L_{n}\right\}: f \in \mathfrak{M}\right\}: L_{n} \subset X\right\}, \\
& \lambda_{n}(\mathfrak{M}, X)=\inf \left\{\inf \left\{\sup \left\{\|f-\Lambda f\|_{X}: f \in \mathfrak{M}\right\}: \Lambda \subset \mathfrak{L}\left(X, L_{n}\right)\right\}: L_{n} \subset X\right\}, \\
& \Pi_{n}(\mathfrak{M}, X)=\inf \left\{\inf \left\{\sup \left\{\|f-\Lambda f\|_{X}: f \in \mathfrak{M}\right\}: \Lambda \subset \mathfrak{L}^{\perp}\left(X, L_{n}\right)\right\}: L_{n} \subset X\right\},
\end{aligned}
$$

где $\mathfrak{L}\left(X, L_{n}\right)$ - множество всех линейных ограниченных операторов из $X$ в $L_{n}, \mathfrak{L}^{\perp}\left(X, L_{n}\right)$ - подмножество проекторов из $\mathfrak{L}\left(X, L_{n}\right)$, а внешняя нижняя грань вычисляется по всем подпространствам $L_{n}$ из $X$ размерности $n$. Эти величины соответственно называются колмогоровским, линейньм и проекиионными поперечниками.

Между перечисленными поперечниками выполняются неравенства

$$
d_{n}(\mathfrak{M}, X) \leqslant \lambda_{n}(\mathfrak{M}, X) \leqslant \Pi_{n}(\mathfrak{M}, X) .
$$

Пусть $f(z)$ - произвольная аналитическая в $|z|<1$ функция,

$$
f(z)=\sum_{k=0}^{\infty} c_{k} z^{k}, \quad z=\rho e^{i t}, \quad 0 \leqslant \rho<1,
$$

с конечной нормой пространства Харди $H_{p}, 1 \leqslant p \leqslant \infty$,

$$
\|f\|_{H_{p}}=\lim _{\rho \rightarrow 1-0}\left(\frac{1}{2 \pi} \int_{0}^{2 \pi}\left|f\left(\rho e^{i t}\right)\right|^{p} d t\right)^{1 / p}<\infty .
$$

Хорошо известно [6], что для $f(z) \in H_{p}$ почти везде на окружности $|z|=1$ существуют угловые граничные значения $F(t)=f\left(e^{i t}\right) \in L_{p}, 1 \leqslant p \leqslant \infty$.

Через $F^{(r)}(t)\left(r=0,1, \ldots, F^{(0)}(t) \equiv F(t)\right)$ обозначим граничные значения аналитической функции $f^{(r)}(z)=d^{r} f / d z^{r}$, а через $F_{a}^{(r)}(t)\left(r=0,1, \ldots, F_{a}^{(0)}(t)=F(t)\right)$ - граничные значения аналитической функции $d^{r} f / d t^{r}=d^{r} f\left(\rho e^{i t}\right) / d t^{r}$. При этом

$$
\begin{aligned}
& \frac{d^{r} f}{d z^{r}}=\sum_{k=r}^{\infty} k(k-1) \cdots(k-r+1) c_{k} z^{k-r} \\
& \frac{d^{r} f\left(\rho e^{i t}\right)}{d t^{r}}=\sum_{k=1}^{\infty}(i k)^{r} c_{k} \rho^{k} e^{i k t}, \quad \rho \neq 0 .
\end{aligned}
$$


Во всем дальнейшем изложении положим

$$
\alpha_{k r}=k(k-1) \cdots(k-r+1), \quad k=r, r+1, \ldots, \quad \beta_{k r}=k^{r}, \quad k, r=1,2, \ldots .
$$

Пусть

$$
\mathscr{P}_{n}=\left\{p_{n}(z): p_{n}(z)=\sum_{k=0}^{n} a_{k} z^{k},\left|a_{n}\right| \neq 0\right\}
$$

Величина

$$
E_{n}(f)_{p}:=E\left(f, \mathscr{P}_{n-1}\right)_{H_{p}}=\inf \left\{\left\|f-p_{n-1}\right\|_{H_{p}}: p_{n-1}(z) \in \mathscr{P}_{n-1}\right\}
$$

есть наилучшее приближение функции $f(z) \in H_{p}$ полиномами $p_{n-1}(z) \in \mathscr{P}_{n-1}$.

Если $f(z) \in H_{p}$ имеет непрерывные граничные значения $F(t)$, то их гладкость характеризуем модулем непрерывности $m$-го порядка в $L_{p}$-норме

$$
\begin{gathered}
\omega_{m}(F ; t)_{p}=\sup \left\{\left\|\Delta_{m}(F ; \cdot, u)\right\|_{p}: u \in[0, t]\right\}, 1 \leqslant p \leqslant \infty \\
\Delta_{m}(F ; x, u) \stackrel{\text { def }}{=} \sum_{k=0}^{m}(-1)^{k} C_{m}^{k} F(x+k u) .
\end{gathered}
$$

В частности, из (1) следует, что если $F^{(r)}(t)$ угловое граничное значение производной $f^{(r)}(z) \in H_{2}$, то согласно равенству Парсеваля

$$
\omega_{m}^{2}\left(F^{(r)} ; t\right)_{2}=2^{m} \cdot \sup \left\{\sum_{k=r+1}^{\infty} \alpha_{k r}^{2}\left|c_{k}\right|^{2}(1-\cos (k-r) u)^{m}: u \in[0, t]\right\} .
$$

Для аналитических в единичном круге функций $f(z) \in H_{2}$ в силу уравнения замкнутости справедливо равенство [7, с. 288-290]

$$
E_{n}^{2}(f)_{H_{2}}=\frac{1}{2 \pi} \int_{0}^{2 \pi}|F(t)|^{2} d t-\sum_{k=0}^{n-1}\left|c_{k}\right|^{2}=\sum_{k=n}^{\infty}\left|c_{k}\right|^{2}
$$

Имеет место следующее утверждение.

Tеорема 1. Пусть для аналитической внутри единичного круга функции $f(z)$ ее производные $f^{(r)}(z)$ и $d^{r} f / d t^{r}$ принадлежсат пространству $H_{2}$ и имеют непрерывные граничные значения $F^{(r)}(t)$ и $F_{a}^{(r)}(t)$. Тогда для любых натуральных $m, n, r$ соответственно при $0<h \leqslant \pi /(n-r), r<n u 0 \leqslant h \leqslant \pi / n$ для $1 \leqslant p \leqslant 2$ имеют место неравенства

$$
\begin{gathered}
E_{n}^{2}(f)_{H_{p}} \leqslant\left\{2^{m} \alpha_{n r}^{2} \int_{0}^{h}(1-\cos (n-r) t)^{m} d t\right\}^{-1} \cdot \int_{0}^{h} \omega_{m}^{2}\left(F^{(r)}, t\right)_{2} d t, \quad r<n \\
E_{n}^{2}(f)_{H_{p}} \leqslant\left\{2^{m} \beta_{n r}^{2} \int_{0}^{h}(1-\cos n t)^{m} d t\right\}^{-1} \cdot \int_{0}^{h} \omega_{m}^{2}\left(F_{a}^{(r)}, t\right)_{2} d t
\end{gathered}
$$

которие являются точными в том смисле, что существует функиия $f_{0}(z) \in H_{p}$, $1 \leqslant p \leqslant 2$, обращающая (4) и (5) в равенства. 
ДоКАЗАТЕЛЬСтво. Равенства (4) и (5) доказываются одинаково, и потому достаточно доказать (4). Из определения модуля непрерьвности (2) следует, что

$$
\begin{aligned}
\int_{0}^{h} \omega_{m}^{2}\left(F^{(r)}, t\right)_{2} d t & \geqslant \int_{0}^{h}\left\|\Delta_{m}\left(F^{(r)}, \cdot, t\right)\right\|_{2}^{2} d t=2^{m} \sum_{k=r+1}^{\infty} \alpha_{k r}^{2}\left|c_{k}\right|^{2} \int_{0}^{h}(1-\cos (k-r) t)^{m} d t \\
& \geqslant 2^{m} \sum_{k=n}^{\infty} \alpha_{k r}^{2}\left|c_{k}\right|^{2} \int_{0}^{h}(1-\cos (k-r) t)^{m} d t
\end{aligned}
$$

Функция

$$
\psi(k)=\alpha_{k r}^{2} \int_{0}^{h}(1-\cos (k-r) t)^{m} d t
$$

для значений $k \geqslant n>r$ является возрастающей, поскольку

$$
\psi^{\prime}(k)=\frac{\alpha_{k r}^{2}}{k-r} h(1-\cos (k-r) h)^{m}+\alpha_{k r}^{2}\left\{\sum_{s=0}^{r-1} \frac{2}{k-s}-\frac{1}{k-r}\right\} \int_{0}^{h}(1-\cos (k-r) t)^{m} d t \geqslant 0 .
$$

Поэтому $\min \{\psi(k): n \leqslant k<\infty\}=\psi(n)$ и из $(6)$ получим

$$
\int_{0}^{h} \omega_{m}^{2}\left(F^{(r)}, t\right)_{2} d t \geqslant 2^{m} \alpha_{n r}^{2} \int_{0}^{h}(1-\cos (n-r) t)^{m} d t \cdot \sum_{k=n}^{\infty}\left|c_{k}\right|^{2}
$$

Отсюда, учитывая равенство (3), запишем

$$
E_{n}^{2}(f)_{H_{2}} \leqslant\left\{2^{m} \alpha_{n r}^{2} \int_{0}^{h}(1-\cos (n-r) t)^{m} d t\right\}^{-1} \cdot \int_{0}^{h} \omega_{m}^{2}\left(F^{(r)}, t\right)_{2} d t
$$

Теперь для завершения доказательства остается учесть, что в силу неравенства Гёльдера

$$
E_{n}^{2}(f)_{H_{p}} \leqslant E_{n}^{2}(f)_{H_{2}}, \quad 1 \leqslant p \leqslant 2,
$$

и оценка (4) сразу следует из соотношений (7) и (8). С другой стороны, используя равенство $E_{n}^{2}\left(z^{n}\right)_{H_{p}}=1, p \geqslant 1$ (см. [8, лемма 3]), и подставляя $f_{0}(z)=z^{n} \in H_{p}$ в $(4)$, получаем требуемое утверждение.

Пусть $\Phi(u)$ - произвольная положительная возрастающая при $u \geqslant 0$ функция такая, что

$$
\lim _{u \rightarrow 0} \Phi(u)=\Phi(0)=0 .
$$

Для любых целых неотрицательных $m$ и $r$ определим в $H_{2}$ классы функций

$$
\begin{aligned}
W_{m}^{r}(\Phi) & =\left\{f(z): f^{(r)}(z) \in H_{2}, \int_{0}^{u} \omega_{m}^{2}\left(F^{(r)}, t\right)_{2} d t \leqslant \Phi^{2}(u), 0<u<\pi\right\} \\
W_{a, m}^{r}(\Phi) & =\left\{f(z): f_{a}^{(r)}(z) \in H_{2}, \int_{0}^{u} \omega_{m}^{2}\left(F_{a}^{(r)}, t\right)_{2} d t \leqslant \Phi^{2}(u), 0<u<\pi\right\} .
\end{aligned}
$$

Положим

$$
(1-\cos n t)_{*}^{m}= \begin{cases}(1-\cos n t)^{m}, & \text { если } n t \leqslant \pi, \\ 2^{m}, & \text { если } n t>\pi .\end{cases}
$$

Результаты теоремы 1 позволяют вычислить точные значения перечисленных выше поперечников на классах $W_{m}^{r}(\Phi)$ и $W_{a, m}^{r}(\Phi)$ в пространстве $H_{2}$. 
Tеорема 2. Пусть функиия $\Phi(u)$ для любого заданного $\mu \in(0,1]$ и для всех $\lambda>0$, $u \in(0, \pi]$, удовлетворяет условию

$$
\Phi^{2}(\mu u) \int_{0}^{\lambda \pi}(1-\cos v)_{*}^{m} d v \leqslant \Phi^{2}(\lambda u) \int_{0}^{\mu \pi}(1-\cos v)^{m} d v .
$$

Тогда для любых натуральных $n, m$ и $г$ справедливы равенства

$$
\begin{gathered}
\gamma_{n}\left(W_{m}^{r}(\Phi), H_{2}\right)=\frac{\sqrt{n-r}}{2^{m / 2} \alpha_{n r}}\left\{\int_{0}^{\mu \pi}(1-\cos t)^{m} d t\right\}^{-1 / 2} \Phi\left(\frac{\mu \pi}{n-r}\right), \quad r<n, \\
\gamma_{n}\left(W_{a, m}^{r}(\Phi), H_{2}\right)=\frac{\sqrt{n}}{2^{m / 2} \beta_{n r}}\left\{\int_{0}^{\mu \pi}(1-\cos t)^{m} d t\right\}^{-1 / 2} \Phi\left(\frac{\mu \pi}{n}\right),
\end{gathered}
$$

әде $\gamma_{n}(*)$ - любой из поперечников $d_{n}(*), \lambda_{n}(*), \Pi_{n}(*)$.

ДокАЗАтЕльство. Не уменьшая общности, проведем рассуждения для класса $W_{m}^{r}(\Phi)$. Полагая в (4) $h=\mu \pi /(n-r), r<n$, получим оценку сверху для проекционного поперечника

$$
\begin{aligned}
\Pi_{n}\left(W_{m}^{r}(\Phi), H_{2}\right) & \leqslant \sup \left\{E_{n}(f)_{H_{2}}: f \in W_{m}^{r}(\Phi)\right\} \\
& \leqslant 2^{-m / 2} \alpha_{n r}^{-1}\left\{\int_{0}^{\mu \pi /(n-r)}(1-\cos (n-r) t)^{m} d t\right\}^{-1 / 2} \Phi\left(\frac{\mu \pi}{n-r}\right) \\
& =\frac{\sqrt{n-r}}{2^{m / 2} \alpha_{n r}}\left\{\int_{0}^{\mu \pi}(1-\cos t)^{m} d t\right\}^{-1 / 2} \Phi\left(\frac{\mu \pi}{n-r}\right), \quad r<n .
\end{aligned}
$$

$\mathrm{C}$ целью получения оценки снизу рассмотрим сферу полиномов

$$
S_{n+1}=\left\{p_{n}(z):\left\|p_{n}\right\|_{H_{2}}=\frac{\sqrt{n-r}}{2^{m / 2} \alpha_{n r}}\left\{\int_{0}^{\mu \pi}(1-\cos t)^{m} d t\right\}^{-1 / 2} \Phi\left(\frac{\mu \pi}{n-r}\right)\right\}
$$

и покажем, что она входит в класс $W_{m}^{r}(\Phi)$. Так как

$$
p_{n}^{(r)}(z)=\sum_{k=r}^{n} \alpha_{k r} c_{k} z^{k-r}
$$

то, полагая $p_{n}^{(r)}(t)=p_{n}^{(r)}\left(e^{i t}\right)$ при $t \in[0, \pi /(n-r)]$, согласно равенству Парсеваля имеем

$$
\left\|\Delta_{m}\left(p_{n}^{(r)} ; \cdot, t\right)\right\|_{2}^{2}=2^{m} \sum_{k=r+1}^{n} \alpha_{k r}^{2}\left|c_{k}\right|^{2}(1-\cos (k-r) t)^{m} .
$$

Если учесть неравенства $(1-\cos (k-r) t)^{m} \leqslant(1-\cos (n-r) t)_{*}^{m}$, справедливые для любых $t \geqslant 0, r<k \leqslant n$, и равенство

$$
\left\|p_{n}\right\|_{H_{2}}^{2}=\sum_{k=0}^{n}\left|c_{k}\right|^{2}
$$

то из (13) и (14) следует, что

$$
\omega_{m}^{2}\left(p_{n}^{(r)} ; t\right)_{2} \leqslant 2^{m} \alpha_{n r}^{2}(1-\cos (n-r) t)_{*}^{m}\left\|p_{n}\right\|_{H_{2}}^{2} .
$$

Интегрируя последнее неравенство по отрезку $[0, \pi /(k-r)], k \geqslant r+1$, и учитьвая (12), приходим к соотношению

$$
\begin{aligned}
& \int_{0}^{\pi /(k-r)} \omega_{m}^{2}\left(p_{n}^{(r)} ; t\right)_{2} d t \\
& \quad \leqslant \int_{0}^{(n-r) \pi /(k-r)}(1-\cos v)_{*}^{m} d v\left\{\int_{0}^{\mu \pi}(1-\cos v)^{m} d v\right\}^{-1} \Phi^{2}\left(\frac{\mu \pi}{n-r}\right) .
\end{aligned}
$$


Полагая в (15) $u=\pi /(n-r), \lambda=(n-r) /(k-r)$, с учетом (9) получим

$$
\int_{0}^{\lambda u} \omega_{m}^{2}\left(p_{n}^{(r)} ; t\right)_{2} d t \leqslant \int_{0}^{\lambda \pi}(1-\cos v)_{*}^{m} d v\left\{\int_{0}^{\mu \pi}(1-\cos v)^{m} d v\right\}^{-1} \Phi^{2}(\mu u) \leqslant \Phi^{2}(\lambda u)
$$

а это означает, что $S_{n+1} \subset W_{m}^{r}(\Phi)$. В силу теоремы о поперечнике сферы (см., например, $[9$, с. 254$])$ для колмогоровского поперечника

$$
d_{n}\left(W_{m}^{r}(\Phi), H_{2}\right) \geqslant \frac{\sqrt{n-r}}{2^{m / 2} \alpha_{n r}}\left\{\int_{0}^{\mu \pi}(1-\cos t)^{m} d t\right\}^{-1 / 2} \Phi\left(\frac{\mu \pi}{n-r}\right), \quad r<n,
$$

чем и завершаем доказательство теоремы 2.

СледствиЕ. В условиях теоремы 2 при $\mu=1$ справедливы равенства

$$
\begin{gathered}
\gamma_{n}\left(W_{m}^{r}(\Phi), H_{2}\right)=\left\{\frac{m !(n-r)}{\pi 2^{m}(2 m-1) ! !}\right\}^{1 / 2} \frac{1}{\alpha_{n r}} \Phi\left(\frac{\pi}{n-r}\right), \quad r<n, \\
\gamma_{n}\left(W_{a, m}^{r}(\Phi), H_{2}\right)=\left\{\frac{m ! n}{\pi 2^{m}(2 m-1) ! !}\right\}^{1 / 2} \frac{1}{\beta_{n r}} \Phi\left(\frac{\pi}{n}\right),
\end{gathered}
$$

әде $\gamma_{n}(*)$ - любое из перечисленных выше поперечников.

В заключение отметим, что функции $\Phi(u)$, удовлетворяющие условию (9) теоремы 2 , существуют [10].

\section{СПИСОК ЦИТИРОВАННОЙ ЛИТЕРАТУРЫ}

1. Тайков Л. В. // Матем. заметки. 1977. Т. 22. № 2. С. 285-295. 2. Айнуллоев Н., Тайков Л. В. // Матем. заметки. 1986. Т. 40. №3. С. 341-351. 3. Айнуллоев Н. // Геом. вопросы теории функций и множеств. Калинин, 1986. С. 91-101. 4. Вакарчук С. Б. // Укр. матем. ж. 1989. Т. 41. №6. С. 799-803. 5. Шабозов М.Ш., Шабозов О. Ш. // Докл. АН Респ. Таджикистан. 1997. Т. 40. № 11. С. 9-12. 6. Привалов И. И. Граничные свойства аналитических функций. М.: Гостехиздат, 1950. 7. Смирнов В.И., Лебедев Н. А. Конструктивная теория функций комплексного переменного. М.-Л.: Наука, 1964. 8. Двейрин М. З., Чебаненко И. В. О полиномиальной аппроксимации в банаховых пространствах аналитических функций. Киев.: Наукова думка, 1983. 9. Корнейчук Н. П. Экстремальные задачи теории приближения. М.: Наука, 1976. 10. Тайков Л. В. // Матем. заметки. 1979. Т. 25. № 2. С. 217-223.

(М. Ш. Шабозов) Хорогский государственный университет 